\title{
Cytokeratin 14 as a marker of squamous differentiation in transitional cell carcinomas
}

\author{
P Harnden, J Southgate
}

\begin{abstract}
The presence of squamous differentiation in transitional cell carcinomas has been variably related to prognosis and response to radiotherapy. This study sought to establish whether cytokeratin (CK) 14, normally expressed in the basal cells of squamous epithelium, could be used as a reliable marker of the emergence of a squamous phenotype in transitional cell carcinomas. In a series of 42 tumours, CK14 was expressed in areas of squamous morphology, whereas CK20 identified continuing urothelial differentiation. Furthermore, focal positivity for CK14 was present in a proportion of tumours with no morphological evidence of squamous differentiation, suggesting that it is a more sensitive marker of phenotypic switch. Investigation of $\mathrm{CK}$ subtypes may be more powerful than morphology alone in clinical studies of transitional cell carcinomas as CK expression profiles have been related to treatment response in other tumour types.

(F Clin Pathol 1997;50:1032-1033)
\end{abstract}

Keywords: cytokeratins; squamous differentiation; transitional cell carcinomas

The wide acceptance of cytokeratins $(\mathrm{CK})$ as excellent epithelial cell markers is largely related to the differentiation specific patterns of expression shown by individual $\mathrm{CK}$ isotypes and the conservation of these patterns following neoplastic transformation.

The expression of one CK isotype (CK20) is restricted to terminally differentiated cells in normal urothelium but is extended to all layers in dysplasia ${ }^{2}$ and some invasive transitional cell carcinomas. ${ }^{3}$ However, CK expression patterns may be influenced by proliferation and differentiation change. ${ }^{4}$ Urothelium shows a propensity for squamous redifferentiation in inflammatory or regenerative states. The possibility of squamous differentiation in transitional cell carcinomas is well recognised and its presence is associated with poorer prognosis ${ }^{5}$ and lack of response to radiotherapy by some ${ }^{6}$ but not others. $^{7}$

The identification of squamous differentiation may be clinically important but can be subjective. The presence of keratin, referring to extracellular generally lamellated material (cornification), or of intercellular bridges are recognised as markers of squamous differentiation. The potential for cornification and intercellular bridging reduces as malignant squamous cells dedifferentiate so that difficulties in identifying the squamous phenotype on routinely stained sections may lead to inconclusive results in clinical studies. ${ }^{67}$

The CK14 isotype is expressed in the basal compartment of all stratified squamous epithelia. ${ }^{4}$ Squamous redifferentiation by human urothelial cells in vitro, induced by retinoid deficiency in the growth medium, is accompanied by de novo expression of CK14. We therefore sought to determine whether immunohistochemical analysis of CK14 expression may serve as a sensitive marker of squamous change in transitional cell carcinomas.

\section{Materials and methods}

Forty two consecutive invasive tumours with an in situ component (to confirm their primary nature) were included, 37 arising from the urinary bladder, four from the ureter, and one from the renal pelvis. The tumours comprised pure squamous cell carcinomas with keratin formation $(n=4)$, transitional cell carcinomas with no other lines of differentiation by light microscopy (pure transitional cell carcinomas) $(n=19)$, and transitional cell carcinomas with either definite areas of squamous differentiation or areas suggestive of squamous differentiation $(n=19)$. Definite squamous differentiation was identified by the presence of cornification, intercellular bridges or both. Cells with cytoplasm more eosinophilic than that of transitional cells and forming cohesive sheets were considered to be suggestive of squamous differentiation. Eight tumours were of moderate grade, the remainder were poorly differentiated.

Anti-CK20 antibody (clone CK20.8) was purchased from Dako Ltd (High Wycombe, Bucks, UK) and anti-CK14 antibody (clone LL002) from Novocastra Laboratories (Newcastle upon Tyne, UK). Paraffin wax embedded sections ( $4 \mu \mathrm{m}$ thick) were dewaxed and rehydrated by transfer through graded alcohols. Endogenous peroxidase activity was blocked by placing the sections in $0.3 \%$ (vol $/ \mathrm{vol}$ ) $\mathrm{H}_{2} \mathrm{O}_{2}$ in methanol for 30 minutes at room temperature before washing. To expose antigens masked by processing the tissue, sections for CK20 labelling were treated with $0.05 \%$ trypsin in $0.1 \%$ calcium chloride, $\mathrm{pH}$ 7.8, for 10 minutes, and those for CK14 labelling were placed in boiling $0.01 \mathrm{M}$ citrate buffer, $\mathrm{pH}$ 6.0, and cooked at pressure for 90 seconds. Slides were then washed to stop further antigen retrieval. Non-specific binding sites were blocked by incubation of sections in 
Table 1 Labelling patterns

\begin{tabular}{lll}
\hline Morphology & CK20 expression & CK14 expression \\
\hline Squamous cell carcinomas & All negative & All positive \\
Pure transitional cell carcinomas & $13 / 19$ positive & $6 / 13$ clusters of positive cells \\
& $6 / 19$ negative & $7 / 13$ negative \\
& & $3 / 6$ clusters of positive cells \\
& & $3 / 6$ negative \\
Transitional cell carcinomas with & $16 / 19$ positive & $15 / 16$ focally positive \\
focal squamous differentiation & $3 / 19$ negative & $1 / 16$ negative \\
& & $3 / 3$ focally positive
\end{tabular}

$20 \%$ ( $\mathrm{vol} / \mathrm{vol}$ ) normal swine serum in $0.1 \%$ sodium azide in Tris buffered saline for 10 minutes. The sections were incubated with primary antibody for 60 minutes and the bound antibody amplified and visualised using the Dako streptavidin ABC complex/HRP duet, mouse/rabbit kit used according to the manufacturer's instructions. Sections were developed in diaminobenzidine and enhanced on $0.4 \%$ copper sulphate for five minutes, counter stained in Harris's haematoxylin, blued in Scott's tap water, dehydrated, cleared, and mounted. Normal urothelium and squamous epithelium served as positive controls for CK20 and CK14, respectively. Negative controls, from which primary antibody was omitted, were performed for all specimens.

\section{Results}

Labelling patterns are shown in table 1. Overall, $93 \%$ of tumours expressed either or both markers.

All pure squamous cell carcinomas were positive for CK14 and negative for CK20. Expression of CK14 was dysregulated in these carcinomas as positivity remained even in areas of cornification, unlike in normal squamous epithelium where CK14 expression was confined to the basal layer.

In the transitional cell carcinomas, CK14 was positive in all areas of definite squamous differentiation identified morphologically (fig 1 ), as well as in areas suggestive of squamous differentiation in all but one case, which showed widespread CK20 expression. Immunocytochemistry for CK14 further detected clusters of cells with a squamous phenotype in $47 \%$ of the tumours with no morphological evidence of squamous differentiation.

\section{Discussion}

We have confirmed that CK14 is expressed in pure squamous cell carcinomas and in transitional cell carcinomas within morphological areas of squamous differentiation, while CK20 is associated with continuing urothelial differentiation. Dual expression of these markers may be useful in confirming the primary nature of a poorly differentiated carcinoma, as other common carcinomas that express CK20, such as gastrointestinal primaries, are not associated with squamous differentiation. On the other hand, the demonstration of CK14 but not CK20 positivity should direct investigations towards the usual sites of primary squamous cell carcinomas.

CK14 was expressed in $71 \%$ of transitional cell carcinomas in the current series although only $50 \%$ had features suggestive or diagnostic

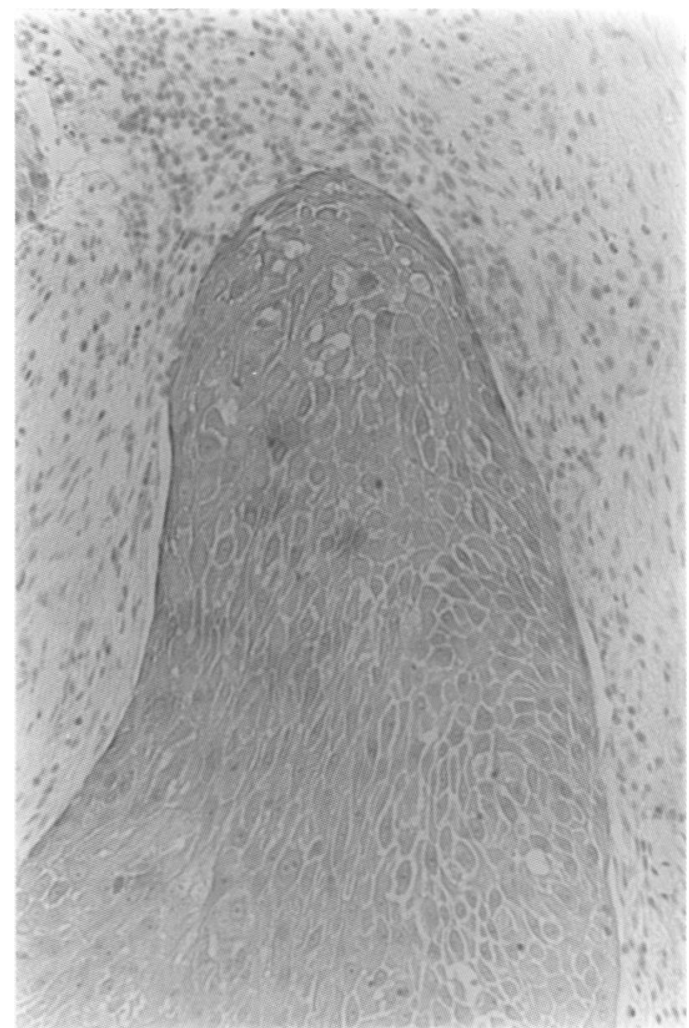

Figure 1 Squamous differentiation, positive for CK14, showing intercellular bridging (original magnification $\times 200$ ).

of squamous differentiation. This is in keeping with the observation that CK14 and its obligate heterodimer $\mathrm{CK} 5$ are the first to be expressed in post-gastrulation mouse embryo skin, before evidence of stratification appears. ${ }^{9}$ Therefore, CK14 expression may be a sensitive marker within urothelial tumours of a switch towards a squamous phenotype. Investigation of CK profiles rather than reliance on morphology alone may be of value in clinical studies as it has been shown that chemotherapeutic agents could modify CKs, and that the drug resistance phenotype in vitro correlates with the expression of specific CK subtypes. ${ }^{10}$

1 Moll R. Cytokeratins in the histological diagnosis of malignant tumors. Int $\mathcal{F}$ Biol Markers 1994;9:63-9.

2 Harnden P, Eardley I, Joyce AD, Southgate J. Cytokeratin 20 as an objective marker of urothelial dysplasia. Br $\mathcal{Z}$ Urol 1996;78:870-5.

3 Moll R, Lowe A, Laufer J, Franke WW. Cytokeratin 20 in human carcinomas. A new histodiagnostic marker detected by monoclonal antibodies. Am $\mathcal{F}$ Pathol 1992;140:427-47.

4 Cooper D, Schermer A, Sun TT. Classification of human epithelia and their neoplasms using monoclonal antibodies epithelia and their neoplasms using monoclonal antibodies to keratins: strategies,
Invest $1985 ; 52: 243-56$.

5 Tannenbaum SI, Carson CC, Tatum A, Paulson DF Squamous carcinoma of urinary bladder. Urology 1983;22 597-9.

6 Jenkins BJ, Martin JE, Baithun SI, Zuk RJ, Oliver RT, Blandy JP. Prediction of response to radiotherapy in invasive bladder cancer. Br $\mathcal{F}$ Urol 1990;65:345-8.

7 Vale JA, A'Hern RP, Liu K, Hendry WF, Whitfield HN, Plowman PN, et al. Predicting the outcome of radical Plowman PN, et al. Predicting the outcome of radical radiotherap $48-51$.

8 Southgate J, Hutton KA, Thomas DF, Trejdosiewicz LK. Normal human urothelial cells in vitro: proliferation and induction of stratification. Lab Invest 1994;71:583-94.

9 Byrne C, Tainsky M, Fuchs E. Programming gene expression in developing epidermis. Development 1994;120 2369-83.

10 Cress AE, Dalton WS. Multiple drug resistance and intermediate filaments. Cancer Met Rev 1996;15:499-506. 